Journal of
Universal Science
Online

\title{
Early corrective surgery may prevent Baroreflex impairment in congenital heart diseases
}

\section{Domonkos CSEH ${ }^{1}$, Adrienn SÁRKÖZI ${ }^{1}$ and Alexandra PINTÉR ${ }^{1, *}$}

\author{
${ }^{1}$ Institute of Human Physiology and Clinical Experimental Research, Semmelweis University, Hungary \\ *Corresponding Author; e-mail: alexandra.pinter@med.semmelweis-univ.hu
}

Received: 28 October 2014 / Revised: 25 November 2014 / Accepted: 14 December 2014

Keywords: Baroreflex-sensitivty, tetralogy of Fallot, transposition of the great arteries, hypoxia.

Abbreviations:
ANOVA - analysis of variance
BRS - baroreflex-sensitivity
$\mathrm{DBPb}$ - diastolic blood pressure at the brachial artery
DBPc - diastolic blood pressure at the carotid artery
HR - heart rate
NYHA - New York Heart Association
OSAS - obstructive sleep apnoe syndrome
$\mathrm{PPb}$ - pulse pressure at the brachial artery

\author{
PPc - pulse pressure at the carotid artery \\ RRI - RR interval \\ $\mathrm{SBP}$ - systolic blood pressure \\ $\mathrm{SBPb}$ - systolic blood pressure at the brachial artery \\ SBPc - systolic blood pressure at the carotid artery \\ TGA - transposition of the great arteries \\ ToF - tetralogy of Fallot
}

\begin{abstract}
Arrhythmias in tetralogy of Fallot (ToF) and transposition of the great arteries (TGA) could be the consequence of reduced baroreflex-sensitivity (BRS). Hypoxia until the first surgical correction in these patients may impair BRS. We aimed to compare the BRS of ToF, TGA and control subjects and test the effect of the timing of operation on BRS.

19 patients with ToF, 22 patients with TGA and 19 healthy controls were enrolled. Carotid pulse pressure (PPc) was similar in ToF and TGA patients but lower in controls. BRS was lower in the ToF group compared to the control or to the TGA groups. The first operation was performed later in patients with ToF than in patients with TGA. Adjustment for PPc attenuated the difference in BRS between $\mathrm{ToF}$ and control subjects. Adjustment for age at corrective surgery abolished the difference in BRS between the ToF and the TGA groups.

In ToF patients, reduced BRS could be explained by the stiffening of central arteries and the later corrective surgery. Earlier surgical correction may prevent irreversible deterioration of baroreflex-function and arrhythmia development in ToF patients.
\end{abstract}

\section{Introduction}

Tetralogy of Fallot (ToF) and transposition of the great arteries (TGA) are complex congenital heart diseases. These abnormalities are supposed to be the consequence of erroneous development of the aorticopulmonary septum [1]. Surgical correction results in good long-term outcome, however, arrhythmia related cardiac events are wellrecognized complications [2,3]. The mechanism of arrhythmia development is not entirely clear in these patient groups.

Arterial baroreflex plays an essential role in cardiac autonomic regulation [4]. Impaired baroreflex-function shifts the balance towards 
sympathetic overactivity and predisposes to rhythmic disturbances [5]. Baroreflexsensitivity (BRS) is an accepted measure of baroreflex-function [6]. In post-myocardial infarction patients decreased BRS could identify subjects at high risk for arrhythmic events [7] and depressed BRS is an independent predictor of total cardiac mortality [8].

We have found reduced BRS in ToF patients, in line with earlier findings $[9,10]$. In contrast, baroreflex-function was preserved in TGA patients according to our results [11]. Nevertheless, our TGA group was considerably younger than the examined ToF groups.

\section{Material and methods}

\section{Subjects}

Nineteen ToF patients and twenty-two ageand gender-matched TGA patients who were attended the Gottsegen György Hungarian Institute of Cardiology, Budapest were recruited into this study. According to New York Heart Association (NYHA) functional classification, all patients were in class I. Patients with hypertension, diabetes mellitus, clinical instability within the preceding two months, permanent pacing, atrial fibrillation or more than two ectopic beats/min were

\section{Protocol}

Participants were studied in the early afternoon in a quiet room with a controlled temperature of $22 \pm 1{ }^{\circ} \mathrm{C}$. All subjects fasted at least 2 hours prior to the measurements and refrained from drinking alcohol- or caffeinecontaining beverages and from strenous exercise for at least 24 hours before the study.

\section{ECG and blood pressure}

ECG was recorded continuously from the limb lead with the largest $\mathrm{R}$ wave for 10 minutes. Blood pressure was monitored simultaneously and continuously at the radial
Acute hypoxia and chronic intermittent hypoxia was shown to impair baroreflexfunction $[12,13]$. ToF and TGA patients suffer from chronic hypoxia until the corrective surgical intervention.

The first aim of this study was to compare the baroreflex-sensitivity of age- and gendermatched ToF and TGA patients and control subjects. We also aimed to examine whether the timing of the first corrective surgery affects baroreflex-function and differences at the age of repair may explain the inhomogenities of BRS found in these patient groups.

excluded. All of our TGA patient went through atrial switch (Senning) procedures. ToF patients underwent various palliative operations and anatomical repairs. Nineteen age- and gender-matched control subjects were also studied.

All participants and the parents of minors gave written informed consent before taking part in the measurements. The study was approved by the Ethical Committee of the Semmelweis University.

After the arrival, the subjects were equipped with the recording devices, then they rested in the supine position for about 15 minutes until the stabilization of heart rate and mean blood pressure values. Consecutively, a 10-minutelong spontaneous baroreflex-sensitivity measurement was carried out.

artery by an automated tonometric device (Colin CBM-7000, Colin Corporation, Komaki, Japan). Radial blood pressure was calibrated with the same device by systolic 


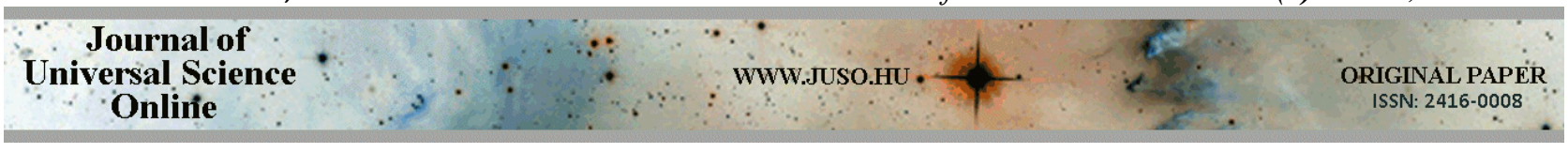

and diastolic blood pressure values measured over the upper arm with a separate oscillometric cuff. The servo-reset function of the Colin system was turned off during the acquisition period.

Blood pressure at the left common carotid

$B R S$

The changes of heart rate induced by spontaneous fluctuation of blood pressure were examined by the sequence method [6]. During the determination of BRS the WinCPRS software (Absolute Aliens Oy, Turku, Finland) detected the $\mathrm{R}$ waves and generated RR interval (RRI) and radial SBP time series, then identified sequences in

\section{Statistical analysis}

Statistical analyses were performed with IBM SPSS v. 22 (IBM Corporation, Somers, New York, USA) Data were expressed as mean \pm SD. Differences between the three groups were examined by one-way analysis of artery was measured by applanation tonometry (SPT-301, Millar Instruments, Houston, TX, USA). The recorded pulse wave was calibrated by brachial diastolic and mean blood pressures measured by oscillometric method [14].

which SBP and RRI concurrently increased over three or more consecutive beats. The lowest accepted change was $1 \mathrm{mmHg}$ for radial SBP and $5 \mathrm{~ms}$ for RRI. The slope of the fitted regression line characterized baroreflex function within the sequences. The slopes of the sequences were averaged producing BRS.

variance (ANOVA) followed by Tukey's post hoc test. Further adjustments were made by binary logistic regression during the paired comparisons of the groups. $\mathrm{P}<0.05$ was considered statistically significant.

\section{Results and discussions}

\section{Results}

Anthropometric and hemodynamic characteristics of the three groups are shown in Table 1. There was no difference in height, weight, SBP, DBP, SBPc, DBPc and HR between the measured groups.

\begin{tabular}{|lcccc|}
\hline Parameters & Controls & ToF & TGA \\
\hline n (male/female) & $19(13 / 6)$ & $19(12 / 7)$ & $22(14 / 8)$ \\
Age (years) & $14 \pm 4$ & $14 \pm 3$ & $14 \pm 3$ \\
Height (m) & $1.59 \pm 0.15$ & $1.58 \pm 0.19$ & $1.59 \pm 0.14$ \\
Weight (kg) & $53 \pm 17$ & $50 \pm 20$ & $49 \pm 15$ \\
SBPb (mmHg) & $110 \pm 12$ & $117 \pm 14$ & $116 \pm 10$ \\
DBPb (mmHg) & $66 \pm 11$ & $62 \pm 7$ & $62 \pm 7$ \\
PPb (mmHg) & $44 \pm 8$ & $58 \pm 12 \ddagger$ & $54 \pm 9 \dagger$ \\
HR (bpm) & $78 \pm 11$ & $78 \pm 9$ & $78 \pm 11$ \\
SBPc (mmHg) & $103 \pm 11$ & $112 \pm 15$ & $110 \pm 12$ \\
DBPc (mmHg) & $67 \pm 12$ & $63 \pm 7$ & $62 \pm 8$ \\
PPc (mmHg) & $36 \pm 7$ & $49 \pm 12 \ddagger$ & $48 \pm 12 \dagger$ \\
\hline
\end{tabular}

Table 1. Anthropometric and hemodynamic parameters of ToF, TGA and control subjects One-way ANOVA with Tukey’s post hoc test $\$$ ToF vs. Controls $p<0.01 ; \uparrow$ TGA vs. Controls $p<0.01$ 
Brachial and carotid pulse pressure $(\mathrm{PPb}$ and $\mathrm{PPc}$, respectively) were significantly higher in ToF patients than in controls $(58 \pm 12$ vs. $44 \pm$ $8 \mathrm{mmHg}, \mathrm{p}<0.01$, and $49 \pm 12$ vs. $36 \pm 7$ $\mathrm{mmHg}, \mathrm{p}<0.01$, respectively).
Similar difference was found between TGA patients and controls $(54 \pm 9$ vs. $44 \pm 8$ $\mathrm{mmHg}, \mathrm{p}<0.01$, and $48 \pm 12$ vs. $36 \pm 7$ $\mathrm{mmHg}, \mathrm{p}<0.01$, respectively).

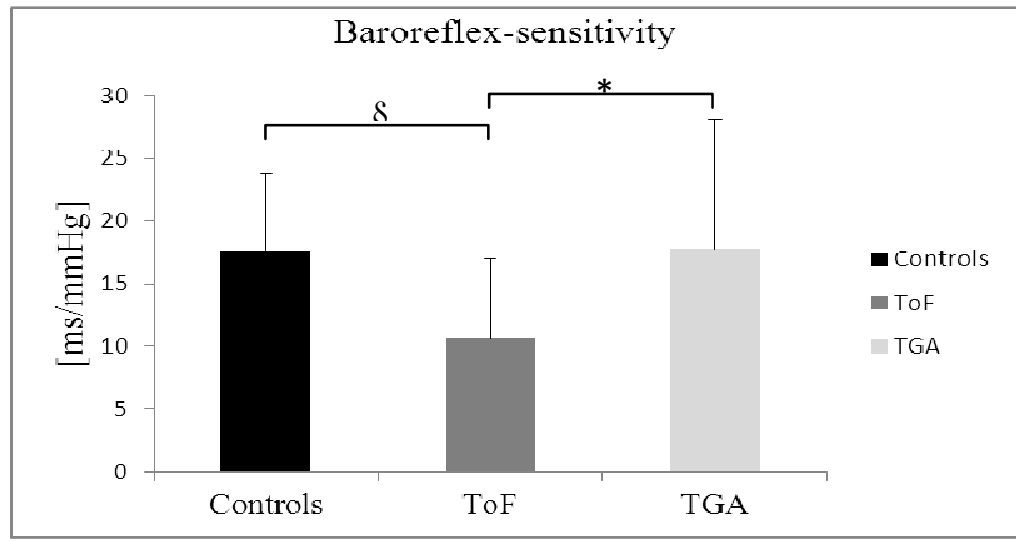

Fig 1. Baroreflex-sensitivity of ToF, TGA and control subjects.

One-way ANOVA with Tukey's post hoc test $\S$ ToF vs. Controls $p=0.024 ; *$ ToF vs. TGA $p=0.016$

There was no difference between the patient groups regarding $\mathrm{PPb}$ and PPc. Data on BRS and the timing of corrective surgery are presented in Figure 1 and Figure 2. BRS was significantly lower in the ToF group compared with the control $(10.62 \pm 6.36$ vs.
$17.61 \pm 6.18 \mathrm{~ms} / \mathrm{mmHg}, \mathrm{p}=0.024)$ or with the TGA group $(10.62 \pm 6.36$ vs. $17.77 \pm$ $10.24 \mathrm{~ms} / \mathrm{mmHg}, \mathrm{p}=0.016)$. We found no difference between the TGA and control group in BRS. Adjusted analyses are shown in Table 2.

\begin{tabular}{|c|c|c|c|c|c|c|}
\hline \multirow[t]{2}{*}{ Model } & \multicolumn{2}{|c|}{ ToF vs. Control } & \multicolumn{2}{|c|}{ TGA vs. Control } & \multicolumn{2}{|c|}{ ToF vs. TGA } \\
\hline & $\begin{array}{l}\text { Regression } \\
\text { coefficient }\end{array}$ & $\mathbf{p}$ & $\begin{array}{c}\text { Regression } \\
\text { coefficient }\end{array}$ & $\mathbf{p}$ & $\begin{array}{l}\text { Regression } \\
\text { coefficient }\end{array}$ & $\mathbf{p}$ \\
\hline \multicolumn{7}{|l|}{ BRS } \\
\hline 1: age + gender & -0.195 & $<0.01$ & 0.007 & 0.867 & -0.110 & 0.024 \\
\hline 2: $1+\mathbf{P P b}$ & -0.184 & 0.042 & -0.016 & 0.733 & -0.121 & 0.021 \\
\hline 3: $1+$ PPc & -0.140 & 0.091 & -0.020 & 0.683 & -0.111 & 0.025 \\
\hline 4: $1+$ age at correction & & & & & -0.092 & 0.303 \\
\hline \multicolumn{7}{|l|}{ Age at correction } \\
\hline 1: age + gender & & & & & 3.278 & $<0.01$ \\
\hline
\end{tabular}

Table 2. Paired comparisons of the groups: adjusted analyses. Binary logistic regression.

After adjustment for age and gender, the first surgical correction was performed significantly later in patients with ToF than in patients with TGA $(2.53 \pm 1.19$ vs. $1.06 \pm$ 0.21 years, $p<0.01$ ). After adjustment for age and gender, the difference in BRS was significant between ToF and control group $(p$ $<0.01)$. This significant difference was not affected by further adjustment for $\mathrm{PPb}(\mathrm{p}=$
0.042). However, adjustment for age, gender and PPc attenuated the difference $(p=0.091)$. After adjustment for age and gender, the difference in BRS remained significant between TGA and ToF group $(p=0.024)$. After further adjustment for the age at corrective surgery the difference did not remain significant $(p=0.303)$. 


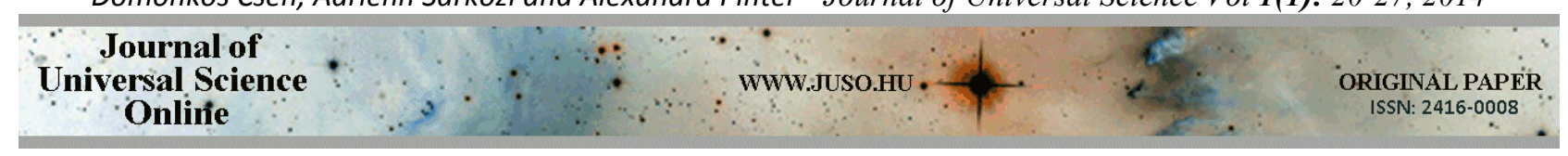

\section{Discussion}

In the present study, we compared the baroreflex-sensitivity of age- and gendermatched ToF and TGA patients and healthy controls. We also examined the influence of the age at first operation on BRS in these patient groups. Confirming earlier findings, baroreflex-function was deteriorated in ToF patients but preserved in the TGA group [9,11]. TGA patients underwent surgical correction significantly earlier and after adjustment for age at surgical correction, there was no more difference in BRS between TGA and ToF patients.

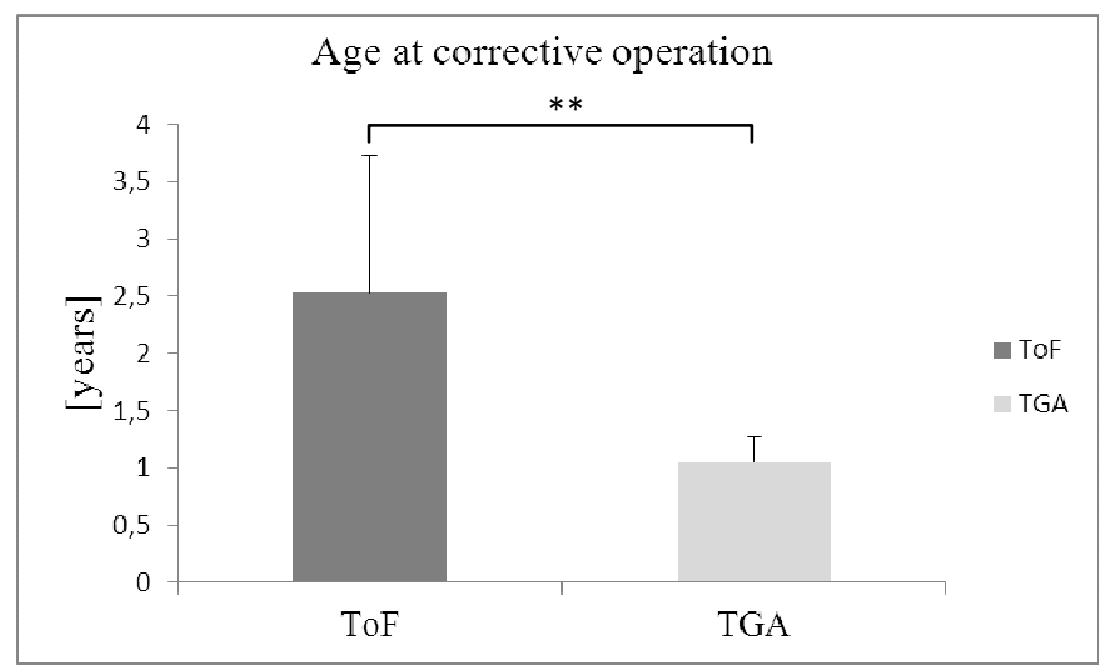

Fig 2. Age at corrective operation of ToF and TGA patients. Binary logistic regression adjusted for age and gender $* * \mathrm{p}<0.01$

Out of the anthropometric and hemodynamic parameters, we found differences between the patient groups and control subjects only in $\mathrm{PPb}$ and PPc. The elevated central pulse pressure in the ToF and TGA group could be the consequence of stiffened central elastic vessels as reported by our group earlier. Embryonic data suggests, that this vascular abnormality may be part of the congenital disease [11,15]. Mechanical properties of the central baroreceptor vascular beds determine the transduction of blood pressure into vessel wall stretch. Decreased elasticity of the common carotid artery may partially explain the impaired baroreflex-function in ToF patients [10]. This hypothesis is supported by our results that adjustment for PPc attenuated the significance of the difference in BRS between ToF patients and controls. Moreover, this significant difference was not affected by adjustment for $\mathrm{PPb}$ instead of PPc. This result confirms the phenomenon that stiffness of the central elastic vessels exhibits stronger correlation with PPc than with $\mathrm{PPb}$ [16].

Along with the distensibility of the central elastic vessels, neural components - baroreceptors, afferent nerves, signal-processing in the central nervous system, efferent nerves, cardiac responsiveness - play an important role in the baroreflex regulation. Neural components with intact plasticity may be able to compensate for the reduced mechanical input from the baroreceptor vessel walls.

Long-lasting exposures to hypoxic conditions were shown to lead to neurodevelopmental impairment [17] and may cause irreversible damage of neural structures of the baroreflex arc. Thus, neural components involved in baroreflex regulation are not able to compensate the deteriorated mechanotransduction of blood pressure changes. Additionally, frequent hypoxic spells before the operation may aggravate these harmful effects in ToF patients. Numerous studies 
have shown the deterioration of cardiac vagal regulation due to acute hypoxia and chronic intermittent hypoxia [12,18,19]. Ryan et al examined the BRS of patients with severe obstructive sleep apnoe syndrome (OSAS) during sleep and found considerably decreased BRS. The desaturation index which is the most important index of the severity of intermittent hypoxia in OSAS was the only independent predictor of impaired baroreflex-function. After adjustment for the duration of the hypoxic period the difference in BRS between ToF and TGA patients did not remain significant supporting our hypothesis. On the contrary, decreased carotid distensibility is not connected with impaired baroreflex-function in TGA [11]. These patients were operated

\section{Limitations}

It was not possible to examine the correlations between BRS and the timing of corrective surgical interventions due to the distribution of the data about the duration of the hypoxic period. Regarding these data: (i) in the TGA group, the standard deviation was very low; (ii) in the ToF group, the standard deviation was higher, however, it was caused by some extreme values. Moreover, patients should be examined at the same age at the end of adolescence due to the possible regeneration and maturation of cardiovagal autonomic function from early childhood until the end of puberty [21]. However, ToF and TGA patients with the same timing of surgery were measured in different ages in our investigation. Further studies are needed with

\section{Conclusions}

In line with earlier findings, we found impaired cardiac autonomic regulation in $\mathrm{ToF}$ patients, but preserved baroreflex-function in patients with TGA. In ToF patients, reduced BRS could be explained partially by the stiffening of central arteries. Moreover, later significantly earlier, thus, their hypoxic period was considerably shorter. The early timing of the surgical correction may contribute to the preservation of neural plasticity.

The surgical procedure may cause unavoidable damage of the cardiac autonomic nerves per se [20]. Therefore, our previous and present data suggest that TGA patients due to the shorter hypoxic period - are able to preserve the regenerational capacity of the neural structures of the baroreflex arc [11].

According to our results, rythmic disturbances in TGA are not the consequences of damaged cardiac autonomic regulation. On the other hand, we speculate that earlier appointment for surgical correction of ToF would lower the risk of arrhythmia development.

(i) larger sample size; (ii) larger overlap between the patient groups regarding the timing of the correction; (iii) measurements at the same age to clarify the role of the elapsed time until the corrective surgery.

We do not have data from the preoperative period regarding the baroreflex-sensitivity, therefore we could not ignore the possibility that surgical correction of ToF had caused the severe damage in cardiac autonomic regulation in this patient group. However, the Senning procedure in TGA-patients involves extensive surgical interruption of the cardiac autonomic nerves, which was not reflected by damaged baroreflex-function late after operation.

corrective operation and longer hypoxic period may contribute to the severe damage of the baroreflex arc. Earlier surgical correction may prevent irreversible deterioration of baroreflex-function and arrhythmia development in ToF patients. 


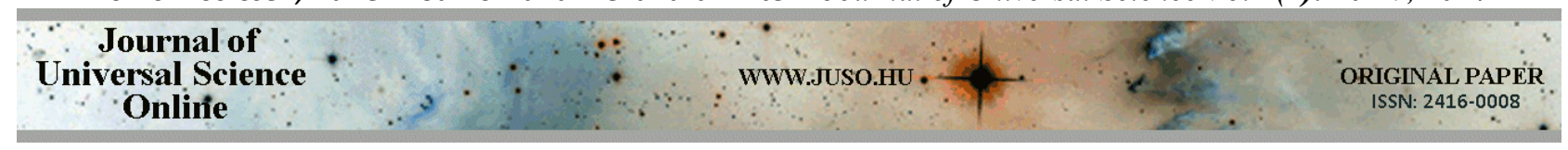

\section{Acknowledgements}

The authors thank Beáta Nagy for the skillful assisstance, Krisztina Kádár MD PhD for the valuable clinical cooperation, Márk Kollai MD PhD for the wise advices and Tamás Horváth MD for the accurate data acquisition and analysis.

\section{References}

1. Rosenquist, T. H.; McCoy, J. R.; Waldo, K. L.; Kirby, M. L., Origin and propagation of elastogenesis in the developing cardiovascular system. Anat Rec 1988, 221 (4), 860-71.

2. Roubertie, F.; Thambo, J. B.; Bretonneau, A.; Iriart, X.; Laborde, N.; Baudet, E.; Roques, X., Late outcome of 132 Senning procedures after 20 years of follow-up. Ann Thorac Surg 2011, 92 (6), 2206-13; discussion 2213-4.

3. Khairy, P.; Aboulhosn, J.; Gurvitz, M. Z.; Opotowsky, A. R.; Mongeon, F. P.; Kay, J.; Valente, A. M.; Earing, M. G.; Lui, G.; Gersony, D. R.; Cook, S.; Ting, J. G.; Nickolaus, M. J.; Webb, G.; Landzberg, M. J.; Broberg, C. S.; (AARCC), A. f. A. R. i. C. C., Arrhythmia burden in adults with surgically repaired tetralogy of Fallot: a multi-institutional study. Circulation 2010, 122 (9), 868-75.

4. Kollai, M.; Jokkel, G.; Bonyhay, I.; Tomcsanyi, J.; Naszlady, A., Relation between baroreflex sensitivity and cardiac vagal tone in humans. Am J Physiol 1994, 266 (1 Pt 2), H21-7.

5. Podrid, P. J.; Fuchs, T.; Candinas, R., Role of the sympathetic nervous system in the genesis of ventricular arrhythmia. Circulation 1990, 82 (2 Suppl), I103-13.

6. Parati, G.; Di Rienzo, M.; Mancia, G., How to measure baroreflex sensitivity: from the cardiovascular laboratory to daily life. J Hypertens 2000, 18 (1), 7-19.

7. La Rovere, M. T.; Pinna, G. D.; Hohnloser, S. H.; Marcus, F. I.; Mortara, A.; Nohara, R.; Bigger, J. T.; Camm, A. J.; Schwartz, P. J.; Infarcton, A. I. A. T. a. R. A. M., Baroreflex sensitivity and heart rate variability in the identification of patients at risk for life-threatening arrhythmias: implications for clinical trials. Circulation 2001, 103 (16), 2072-7.

8. La Rovere, M. T.; Bigger, J. T.; Marcus, F. I.; Mortara, A.; Schwartz, P. J., Baroreflex sensitivity and heart-rate variability in prediction of total cardiac mortality after myocardial infarction. ATRAMI (Autonomic Tone and Reflexes After Myocardial
Infarction) Investigators. Lancet 1998, 351 (9101), 478-84.

9. Davos, C. H.; Davlouros, P. A.; Wensel, R.; Francis, D.; Davies, L. C.; Kilner, P. J.; Coats, A. J.; Piepoli, M.; Gatzoulis, M. A., Global impairment of cardiac autonomic nervous activity late after repair of tetralogy of Fallot. Circulation 2002, 106 (12 Suppl 1), I69-75.

10. Pintér, A.; Horváth, T.; Tóth, A.; Kádár, K.; Kollai, M., Impaired baroreflex function is related to reduced carotid artery elasticity in patients with tetralogy of Fallot. Auton Neurosci 2014, 183, 94-9.

11. Pinter, A.; Laszlo, A.; Mersich, B.; Kadar, K.; Kollai, M., Adaptation of baroreflex function to increased carotid artery stiffening in patients with transposition of great arteries. Clin Sci (Lond) 2007, 113 (1), 41-6.

12. Roche, F.; Reynaud, C.; Garet, M.; Pichot, V.; Costes, F.; Barthélémy, J. C., Cardiac baroreflex control in humans during and immediately after brief exposure to simulated high altitude. Clin Physiol Funct Imaging 2002, 22 (5), 301-6.

13. Lin, M.; Liu, R.; Gozal, D.; Wead, W. B.; Chapleau, M. W.; Wurster, R.; Cheng, Z. J., Chronic intermittent hypoxia impairs baroreflex control of heart rate but enhances heart rate responses to vagal efferent stimulation in anesthetized mice. Am J Physiol Heart Circ Physiol 2007, 293 (2), H997-1006.

14. Van Bortel, L. M.; Balkestein, E. J.; van der Heijden-Spek, J. J.; Vanmolkot, F. H.; Staessen, J. A.; Kragten, J. A.; Vredeveld, J. W.; Safar, M. E.; Struijker Boudier, H. A.; Hoeks, A. P., Non-invasive assessment of local arterial pulse pressure: comparison of applanation tonometry and echo-tracking. $J$ Hypertens 2001, 19 (6), 1037-44.

15. László, A.; Pintér, A.; Horváth, T.; Kádár, K.; Temesvári, A.; Kollai, M.; Studinger, P., Impaired carotid artery elastic function in patients with tetralogy of Fallot. Heart Vessels 2011, 26 (5), 542-8. 


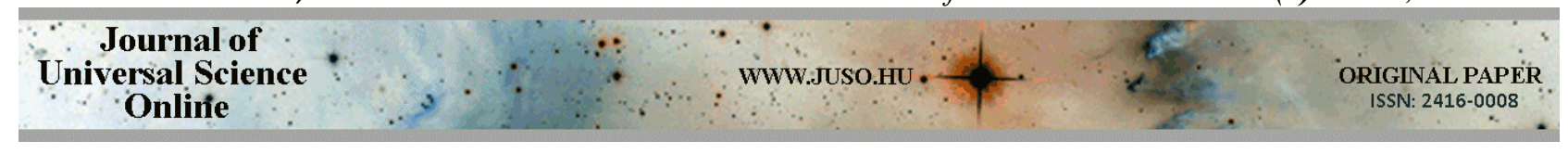

16. Pini, R.; Cavallini, M. C.; Palmieri, V.; Marchionni, N.; Di Bari, M.; Devereux, R. B.; Masotti, G.; Roman, M. J., Central but not brachial blood pressure predicts cardiovascular events in an unselected geriatric population: the ICARe Dicomano Study. J Am Coll Cardiol 2008, 51 (25), 2432-9.

17. Hövels-Gürich, H. H.; Konrad, K.; Skorzenski, D.; Nacken, C.; Minkenberg, R.; Messmer, B. J.; Seghaye, M. C., Long-term neurodevelopmental outcome and exercise capacity after corrective surgery for tetralogy of Fallot or ventricular septal defect in infancy. Ann Thorac Surg 2006, 81 (3), 958-66.

18. Ryan, S.; Ward, S.; Heneghan, C.; McNicholas, W. T., Predictors of decreased spontaneous baroreflex sensitivity in obstructive sleep apnea syndrome. Chest 2007, 131 (4), 1100-7.
19. Lai, C. J.; Yang, C. C.; Hsu, Y. Y.; Lin, Y. N.; Kuo, T. B., Enhanced sympathetic outflow and decreased baroreflex sensitivity are associated with intermittent hypoxia-induced systemic hypertension in conscious rats. J Appl Physiol (1985) 2006, 100 (6), 1974-82.

20. Heragu, N. P.; Scott, W. A., Heart rate variability in healthy children and in those with congenital heart disease both before and after operation. Am J Cardiol 1999, 83 (12), 1654-7.

21. Lenard, Z.; Studinger, P.; Mersich, B.; Kocsis, L.; Kollai, M., Maturation of cardiovagal autonomic function from childhood to young adult age. Circulation 2004, 110 (16), 2307-12. 